\title{
Editorials
}

\section{New technologies and general practice}

\section{INTRODUCTION}

Front-line medicine is arguably under more pressure now than it has ever been. This is at least in part due to a population living longer than ever before with an increasing number of chronic diseases. The management of these conditions is increasingly occurring in the primary and community care setting. If we are to rise to this challenge and continue to provide an NHS true to its founding principles, we need to embrace new technology and the potential benefits it can offer our patients.

\section{THE TECHNOLOGICAL HEALTHCARE REVOLUTION}

Advances in technology are disrupting the world around us, and companies like Uber, Airbnb, Microsoft, Google, and Amazon are utilising these innovations to change the way we live our lives. More and more of our patients want a healthcare system that is personalised and one in which they are empowered to take more control of their health. Developments in 'omics', data analytics, machine learning, artificial intelligence, digital/m-health, advanced technology, and social networking are heralding this personalised, empowered healthcare revolution. However, medical practice has been slow to adapt to this progress. The economist John Maynard Keynes perhaps helped illuminate the problem the medical profession faces when he said:

'The difficulty lies, not in the new ideas, but in escaping from the old ones, which ramify, for those brought up as most of us have been, into every corner of our minds. "

How can we escape from the practices that once served us well, but which have now been superseded by new, disruptive innovations? These advances are not only finding their way into the clinical setting, but are now in our homes and our pockets. Patients are already taking control, with more technology directly available to them to help monitor and manage their health than is available in most general practices. We are moving from an era where doctors were the gatekeepers to (or owners of) healthcare technology to a system of personalised health care. Here, empowered patients have a digital health kit and doctors act as advisors and navigators through the system.

\section{"Patients are already taking control, with more technology directly available to them to help monitor and manage their health than is available in most general practices.}

\section{KEY TECHNOLOGICAL THEMES}

At NHS England we cross paths with many innovative health and technology companies. There are some key technology themes that have emerged that will impact primary and community care.

\section{Connected devices}

From WiFi scales and blood pressure cuffs to the Google Contact Lens that continually measures glucose levels and the CliniCloud Bluetooth stethoscope with a pneumoniadetecting algorithm (https://clinicloud.com), both patients and carers can get involved in managing their health. There is even a nappy that will tweet your smartphone when it needs changing. The 'propeller inhaler' is transforming the treatment of respiratory conditions (https://www.propellerhealth. com). By logging when and where inhalers are being used, patients are connected to their carers and each other. In New York, maps of inhaler use are helping patients to avoid polluted areas and prevent exacerbations of their condition.

\section{Smartphone clip-on and plug-ins}

Many patients now carry more computing power in their pockets than was present in most GP surgeries as little at 10 years ago. The functionality and adaptability of these devices has great potential for transforming health care. The NHS has been supporting Cupris (http://www.cupris.com), a company developing a clip-on that converts the smartphone into an ophthalmoscope and otoscope. Other clip-ons include the AliveCor device (https://www.alivecor.com/en/) for detection of cardiac arrhythmias, a range of dermatoscopes that are heralding the era of teledermatology, and the Dario blood glucose monitor (http://mydario.co.uk). Plug-ins, such as the 'Smart Peak Flow' device (http://www.smartpeakflow.com), are also transforming self-management. Even ultrasound probes can now be plugged into a smartphone, potentially making this a cost-effective option for use in primary care. The Philips Lumify is one example of such a device (https://www.lumify.philips.com/ web/).

\section{Platforms and condition-specific apps}

There are over 160000 apps in the Apple and Android stores reported as being related to health care. However, there is no universally accepted rating system. NHS England, NHS Digital, the National Institute for Health and Care Excellence, and the Medicines and Healthcare products Regulatory Agency are working together through the NHS endorsement programme to start to address this. Other groups such as Orcha (http:// www.orcha.co.uk) have created a health app store', with rated apps. This can be white labelled for local health economies and has been taken up by more than a dozen clinical commissioning groups to date. There are too many excellent health-related apps to cover here, but the 'My mhealth' range of diabetes, asthma, and chronic obstructive pulmonary disease management apps (https://mymhealth.com) is gaining traction in the NHS and My mhealth Limited is one of the NHS England National Innovation

\section{"Many patients now carry more computing power in their pockets than was present in most GP surgeries as little at 10 years ago}




\section{"The challenge we face with the current wave of new technology in primary care is how we can help people to connect with it and feel that it makes a difference to them.}

Accelerator fellows. ${ }^{2}$ On the horizon, an app in development to keep an eye on is Cordio (http://www.cordio-med.com/index.html). This detects changes in a speaker's tone of voice on a mobile phone. Trials are awaiting publication, but early analysis indicates the app can predict admission to hospital with an exacerbation of congestive heart failure up to 10 days in advance of it happening. So you could call your mum in the morning, and she says she's feeling fine, but the app says she needs to take $20 \mathrm{mg}$ more of furosemide!

Prevention is key if we are to deliver a sustainable health and care system moving forward. Platforms like Quealth (http://www. quealth.co) provide a personalised health coach and, using a single number, the $Q$ score can show how you can improve your health. This is freely available to all.

There are many examples of virtual consultationappsand platforms. Increasingly, providers are moving to enhanced offerings beyond the simple video consultation/ doctor-in-your-pocket type approach. For example, HealthTap (https://www.healthtap. com) offers a library of 4.5 billion medically curated questions and answers. Around 10\% of face-to-face consultations can be dealt with via the library and a further $20-30 \%$ via a combination of e-mail/text, telephone, and video consultations. In addition, it offers a professional networking environment between primary and secondary care where questions and answers between professionals can be captured and automatically uploaded to continuing professional development portfolios.

\section{Wearable sensors}

From digital plasters like the Vital Connect (http://www.vitalconnect.com) and Zio Patches (http://www.irhythmtech.com) to ear plugs (http://www.bragi.com), physiological measurements (pulse, respiratory rate, temperature, electrocardiogram), and movement can already be detected, with developments on blood pressure and oxygen saturation ahead. Continuous glucose monitoring patches (https:// www.freestylelibre.co.uk and http://www. dexcom.com/en-GB) are gaining increasing popularity with patients. Also, combinatorial innovations bringing wearable sensors, apps, and a telephone help line are providing an alternative to sleeping tablets in the form of 'Good Sleep' (https://goodsleep.optum. com).

\section{Advanced technology}

Point-of-care testing is set to revolutionise pathology services and patient management in primary care. Systems such as the i-STAT (https://www.abbottpointofcare.com/ products-services/istat-handheld) offer many of the standard chemical pathology tests requested in primary care. Others are integrating electronic patient records, point-of-care testing, and wearable sensors, and providing management pathways, for example, LumiraDx (https://uww.lumiradx. $\mathrm{com} /$ home). There is also a range of medical 'Tricorders' emerging, such as by Scanadu (https://www.scanadu.com) and Cloud DX (http://clouddx.com/). Even a smart digital first-aid kit has recently been launched, called Gale (http://www.19labs.com/).

\section{CHALLENGES AND PRESSURES}

The promise of all this technological innovation appears impressive. However, when previous attempts have been made to roll this out in primary care, the anticipated benefits have not been realised, as shown in the world's largest randomised controlled trial for telehealth. ${ }^{3}$ One of the key findings of the Whole System Demonstrator trial was that patients with 'technology' felt more socially isolated than those without. This is a key consideration as we look to incorporate some of the latest, greatest innovations into patient care pathways. So how can we drive uptake of innovation? In the words of Maya Angelou:

people will forget what you said, people will forget what you did, but people will never forget how you made them feel."

The challenge we face with the current wave of new technology in primary care is how we can help people to connect with it and feel that it makes a difference to them. We need to ensure technology

\section{ADDRESS FOR CORRESPONDENCE}

\section{Antony J Young}

Anglia Ruskin University, Postgraduate Medical Institute, Chelmsford Campus, Bishop Hall Lane, Chelmsford, CM1 1SQ, UK

\section{E-mail: tony.youngdanglia.ac.uk}

increases social inclusion and reduces social isolation.

Finally, we have to consider the pressures that are ever increasing on the front-line in primary care. Some of the technology advances described here look impressive, but if they lead to an increased workload on GPs they will not be adopted. If we are going to deliver the benefits of the latest technology to our patients, we need a new approach. Technology companies need to work with us to show impact across the whole care pathway, where quality of care is improved, patients and their carers feel engaged and empowered, and workload on GPs is reduced. Only then will we be able to deliver our ultimate aim of a high-quality, sustainable NHS free at the point of delivery for generations to come.

\section{Antony J Young,}

Consultant Urological Surgeon, Southend University Hospital, Director of Medical Innovation, Anglia Ruskin University, and National Clinical Lead for Innovation, NHS England.

\section{Competing interests}

The author has declared no competing interests.

\section{Provenance}

Commissioned; not externally peer reviewed.

DOI: 10.3399/bjgp16X688021

\section{REFERENCES}

1. Keynes JM. The general theory of employment, interest and money. London: Macmillan, 1936

2. NHS England. NHS Innovation Accelerator https://www.england.nhs.uk/ourwork/ innovation/nia/ (accessed 10 Nov 2016).

3. The Nuffield Trust. The impact of telehealth and telecare: the Whole System Demonstrator project. http://wnw. nuffieldtrust.org.uk/our-work/projects/impacttelehealth-and-telecare-evaluation-wholesystem-demonstrator-project laccessed 10 Nov 2016) 